\title{
Bilirubin Dependent on UGT1A1 Polymorphisms, Hemoglobin, Fasting Time and Body Mass Index
}

\author{
Carina Rodrigues, MSC, Elísio Costa, PhD, Emília Vieira, MSC, João De Carvalho, MD, \\ Rosário Santos, MSC, Petronila Rocha-Pereira, PhD, Alice Santos-Silva, PhD \\ and Elsa Bronze-da-Rocha, PhD
}

\begin{abstract}
In humans, bilirubin levels are influenced by different factors. This study aims to evaluate the influence of several nongenetic factors (hematologic data, smoking status, alcohol intake, fasting time, physical activity, oral contraceptive therapy and caloric intake) and the genetic contribution of UGTIAI polymorphisms for the bilirubin levels, in a cohort of young women. Hematologic data, bilirubin and screening of TA duplication in the TATA box region of the UGT1A1 gene were performed in 146 young white women. Body mass index (BMI) and body fat were determined, and a questionnaire about fasting time, smoking habits, oral contraceptive therapy, caloric intake and physical activity was performed. Participants were divided into 3 groups according to the tertiles of bilirubin levels. Subjects from the second and third tertile had significant increases in hemoglobin $(\mathrm{Hb})$ concentration, hematocrit, mean cell $\mathrm{Hb}$ and mean cell $\mathrm{Hb}$ concentration compared with those in the first tertile. Red blood cell count was significantly increased in subjects in the third tertile. A significant increased frequency was found for the c.-41_-40dupTA allele in homozygosity for both second and third tertiles. Multiple linear regression analysis showed that the c.-41_-40dupTA allele, $\mathrm{Hb}$, BMI and fasting hours were independent variables associated with bilirubin serum levels. $\mathrm{Hb}$ concentration, fasting time and BMI were identified as nongenetic causes, together with the genetic UGT1A1 polymorphisms, as the main factors associated with variations in bilirubin levels in a healthy female population.
\end{abstract}

Key Indexing Terms: Bilirubin levels; UGT1A1; Genetic and nongenetic factors; Hemoglobin; Gilbert's syndrome. [Am J Med Sci 2011; $\operatorname{XXX}(\mathbf{X X}): 1$.

$B^{i}$ ilirubin is the main metabolic end product of heme breakdown, and it is a key marker of liver and hematologic disorders. Bilirubin itself is a water-insoluble compound that requires glucuronidation by a microsomal enzyme, the uridine diphosphate glucuronosyltransferase (UGT1A1), to be excreted. ${ }^{1}$ UGT1A1 has other endogenous and exogenous substrates, apart from bilirubin, and is implicated in several processes, such as estradiol metabolism and detoxification of

From the Department of Biological Sciences (CR, AS-S, EB-D-R), Biochemistry Laboratory, Faculty of Pharmacy of Oporto University, Porto; Health College of Polytechnic Institute of Bragança (CR), Bragança; Institute of Health Sciences of Portuguese Catholic (EC), Porto; Molecular Genetics Unit (EV, RS), Institute of Medical Genetics Dr. Jacinto Magalhães INSARJ, Porto; Gastroenterology Department (IDC), Centro Hospitalar de Vila Nova de Gaia; Investigation Center for Health Sciences (PR-P), University of Beira Interior, Covilhã; and Institute for Cellular and Molecular Biology (EC, AS-S, EB-D-R), Oporto University, Porto, Portugal.

Submitted January 27, 2011; accepted in revised form April 27, 2011.

This study was supported by PhD grant (SFRH/BD/42791/2007) from Fundação para a Ciência e Tecnologia (FCT) and Fundo Social Europeu (FSE) attributed to Carina Rodrigues.

Correspondence: Elsa Bronze-da-Rocha, PhD, Department of Biological Sciences, Biochemistry Laboratory, Faculty of Pharmacy of Oporto University, Porto, Portugal, Rua Anibal Cunha, 164, 4050-047 Porto, Portugal (E-mail: elsa.rocha@ff.up.pt). potential carcinogens and mutagens. ${ }^{2,3}$ Changes in UGT1A1 activity are therefore able to modify water-soluble bilirubin glucuronidation, drug metabolism.

The UGTIAI gene locus has many variants, and genotyping data describe $>100$ single-nucleotide polymorphisms within the UGT1A1 gene promoter and coding sequences. ${ }^{2,3}$ Wild-type activity is associated with 6 TA repeats in TATA box region of the gene, and the UGTIAl gene expression decreases with increasing number of TA repeats. Moderate to severe unconjugated hyperbilirubinemia results from a severe reduction or from the absence of UGT1A1 activity, occurring in the Crigler-Najjar syndrome type I and type II, respectively, whereas a mild hyperbilirubinemia occurs in the Gilbert's syndrome (GS) ${ }^{4}$ Homozygosity for the c.-41_-40dupTA allele is the most common cause for the Gilbert's syndrome. ${ }^{5}$ Statistics based on incidence and genotype frequencies provided evidence that other inherited and/or acquired factors affect the bilirubin metabolism; however, the UGTIAl gene is known as the major factor. ${ }^{6}$

Bilirubin levels present slight daily changes, increasing during fasting and in adolescence. A slight increase in bilirubin concentration has been reported also during physical exercise, stress or menstruation. ${ }^{7}$ The activity of UGT1A1 can also be enhanced with alcohol intake and by some drugs, ${ }^{8-12}$ or it can be down-regulated during infection and inflammation. ${ }^{12-14}$

Bilirubin in high concentrations is a dangerous metabolite for human health, namely, in newborns that are susceptible to toxicity from unconjugated bilirubin, leading to Kernicterus if it is not immediately treated. ${ }^{15}$ Recent evidences suggest that mild bilirubin levels are strong physiologic antioxidants that may give protection against atherosclerosis, coronary heart disease and inflammation, all known as oxidative stress conditions. ${ }^{16,17}$

In addition to the referred genetic background that regulates serum bilirubin levels, there are other genetic and nongenetic factors that may contribute for bilirubin concentration. Because hemoglobin $(\mathrm{Hb})$ degradation is the main source of bilirubin, it is possible that individual differences in bilirubin concentrations could be due, mainly, to differences in $\mathrm{Hb}$ concentration. Indeed, it was, recently, described that an increase in red cell mass, probably, plays a crucial role in hyperbilirubinemia levels, because they would increase to unexpected higher values in case of GS, ${ }^{18}$ eventually triggering the clinical manifestation of the disease or its worsening.

The aim of this study was to evaluate the influence of $\mathrm{Hb}$ concentration and of several nongenetic factors, such as smoking status, alcohol intake, physical activity, oral contraceptive therapy, fasting time and caloric intake, and the genetic contribution of UGTIAI polymorphisms for the bilirubin levels, by performing a cohort study in young Portuguese women. 


\section{MATERIALS AND METHODS}

\section{Subjects}

We studied a group of 146 randomly selected young white female students $(20.7 \pm 2.6$ years old $)$ from the north of Portugal. All participants gave their informed consent to participate in this study, and those with liver and/or hematologic disorders, chronic or acute infection, under medication, other than contraceptives and with a history of malignancy were excluded from the study.

All participants were asked to respond to a questionnaire, including questions about fasting time, smoking habits and oral contraceptive therapy. To assess physical activity, we applied the Short Form of the International Physical Activity Questionnaire. ${ }^{19}$ Body weight and height were determined by standard anthropometric techniques. Body mass index (BMI) was calculated according to World Health Organization recommendations. Body fat measurement was performed by a bioelectrical impedance system (Model BC532; TANITA, Tokyo).

\section{Samples and Measurements}

Blood samples were collected (ethylenediaminetetraacetic acid as anticoagulant) to obtain whole blood, plasma and buffy coat for DNA extraction. Plasma samples were aliquoted and stored at $-70^{\circ} \mathrm{C}$, until assayed.

Red blood cell count, total and differential white blood cell count, $\mathrm{Hb}$ concentration, hematocrit $(\mathrm{Ht})$ and hematologic indices [mean cell volume, mean cell $\mathrm{Hb}$ $(\mathrm{MCH})$, mean cell $\mathrm{Hb}$ concentration $(\mathrm{MCHC})$ and red cell distribution width] were measured by using an automatic blood cell counter (ABX Micros 60-OT; Horiva-ABX, France). Plasmtic total bilirubin concentration was determined using a colorimetric method (diazotized sulfanilic acid reaction; Roche Diagnostics Co., IN).

\section{Genotype Determination}

All participants were screened for the presence of the TA duplication in the TATA box region of the UGT1A1 gene. This TATA box region was analyzed by polymerase chain reaction (PCR) amplification, using the primers previously described by Bancroft et al, ${ }^{20}$ with the introduction of a fluorochrome label in the reverse primer, for subsequent analysis by automated capillary electrophoresis. In brief, genomic DNA was extracted from blood samples by using the standard salting out method. The TATA box region of UGT1A1 gene was analyzed by PCR amplification. For the reaction mixture, we used the PCR Master Mix (Promega, Madison, WI). To $25 \mu \mathrm{L}$ of this mixture (with dNTPs, DNA Taq polymerase and $\mathrm{MgCl}_{2}$ ) was added 1 $\mu \mathrm{L}$ of each primer $(10 \mathrm{pmol} / \mu \mathrm{L}), 1 \mu \mathrm{L}$ of genomic DNA (100 $\mathrm{ng} / \mu \mathrm{L})$ and water, for a final volume of $50 \mu \mathrm{L}$. The protocol of cycling was performed on a DNA thermocycler GeneAmp PCR System 9700 (Applied Biosystems, Foster City, CA). The first step of denaturation at $95^{\circ} \mathrm{C}$ for 10 minutes was followed by 35 cycles of denaturation at $95^{\circ} \mathrm{C}$ for 1 minute, annealing at $58^{\circ} \mathrm{C}$ for 45 seconds and extension at $72^{\circ} \mathrm{C}$ for 1 minute; and a final extension at $72^{\circ} \mathrm{C}$ for 10 minutes was performed. After PCR, 1 $\mu \mathrm{L}$ of the products was separated by automated capillary electrophoresis (ABI Prism 3130xl Genetic Analyser; Applied Biosystems) and analyzed by GeneScan software v3.7 along with the molecular weight marker GeneScan ROX-500 Size Standard (Applied Biosystems).

\section{Statistical Analysis}

For statistical analysis, we used the Statistical Package for Social Sciences (version 17.0; SPSS, Chicago, IL). Kolmogorov-Smirnov statistics were applied to evaluate sample normality distribution. Continuous variables without normal distribution were log transformed. The participants were divided by tertiles of total bilirubin, and multiple comparisons between the obtained groups were performed by 1-way analysis of variance, supplemented with Tukey's HSD post hoc test. For categorical variables, we used $\chi^{2}$ test or Fisher's exact test to compare groups. Pearson correlation coefficient was applied to evaluate relationships between sets of data. Multiple-regression analysis (stepwise method) was used to find independent variables associated with total bilirubin levels. The genotype of the TATA box polymorphism was coded using 2 dummy variables, one to compare the homozygous for the wild-type allele (reference category) with heterozygous for the c.-41_-40dupTA, and the other to compare the wild-type allele with homozygous for c.-41_-40dupTA. A $P$ value of $<0.05$ was considered statistically significant.

\section{RESULTS}

To analyze the contribution of $\mathrm{Hb}$ and other factors to the bilirubin blood levels, we divided our population of young female participants into 3 groups, according to the tertiles of total bilirubin concentration, the first group $\leq 6 \mu \mathrm{mol} / \mathrm{L}$, the second group between 6 and $9 \mu \mathrm{mol} / \mathrm{L}$ and the third group $\geq 9$ $\mu \mathrm{mol} / \mathrm{L}$.

No differences were observed between the 3 groups when comparing the age of the participants (Table 1). The hematologic studies showed that subjects from the second and third tertile had significantly higher $\mathrm{Hb}$ concentration, $\mathrm{Ht}$, $\mathrm{MCH}$ and $\mathrm{MCHC}$ and lower platelet counts compared with those in the first tertile (Table 1). The subjects from the third tertile maintained the same significant changes, and a significant increase in red blood cell count, compared with the first tertile values. No changes were observed between the second and the third tertile despite the increasing bilirubin concentration. No significant differences were found between groups for total and differential white blood cell counts (Table 1).

The frequency for the c.-41_-40dupTA allele in homozygosity increased from the first to the third tertile because a significant increase was observed between the first and the second tertile, and the frequency for the latter was significantly lower than that observed for the third tertile (Table 1). Allele frequencies of 0.31 for c.-41_-40dupTA allele and of 0.69 for normal allele were obtained in all population, which are in Hardy-Weinberg equilibrium ( $\chi^{2}$ test; $P=0.029$ ). Moreover, when compared, total bilirubin concentration, according to UGT1A1 genotype, a significant and progressive increase was observed, from the $[=]+[=]$ to the homozygous c.-41_-40dupTA allele (Figure 1).

No significant differences were observed between the 3 tertile groups of bilirubin concentration, when considering physical activity, smoking habits, oral contraceptive therapy, body fat, alcohol ingestion and fasting time. However, a significant decrease in BMI was observed in the third tertile compared with the first tertile ( $P=0.009$; Table 2$)$.

Significant positive correlations were found between total bilirubin concentration and $\mathrm{Hb}(r=0.336 ; P<0.0001)$, Ht $(r=0.244 ; P=0.003), \mathrm{MCH}(r=0,276 ; P=0.001)$ and $\mathrm{MCHC}(r=0.326 ; P<0.0001)$.

Multiple linear regression analyses showed that the TATA box gene polymorphism, $\mathrm{Hb}, \mathrm{BMI}$ and fasting time account for approximately $44 \%$ of the variation in serum total bilirubin levels (adjusted $R^{2}=0.439 ; P<0.0001$ ). The model identified the c.-41_-40dupTA allele as the main contributor for total bilirubin variation $(\beta=0.481, P<0.0001$ when in 


\begin{tabular}{|c|c|c|c|c|c|}
\hline & \multirow{2}{*}{$\begin{array}{c}\text { Participants } \\
(n=146)\end{array}$} & \multicolumn{4}{|c|}{ Tertiles of total bilirubin levels $(\mu \mathrm{mol} / \mathrm{L})$} \\
\hline & & $\mathrm{T} 1(\leq 6.0, \mathrm{n}=49)$ & $\mathrm{T} 2(6.0-9.2, \mathrm{n}=49)$ & $\mathrm{T} 3(\geq 9.2, \mathrm{n}=48)$ & $P^{a}$ \\
\hline Age (yr) & $20.5(2.3)$ & $20.9(2.8)$ & $20.3(2.1)$ & $20.4(2.0)$ & 0.332 \\
\hline \multicolumn{6}{|l|}{ Blood count } \\
\hline $\mathrm{RBC}\left(\times 10^{12} / \mathrm{L}\right)$ & $4.6(0.3)$ & $4.5(0.2)$ & $4.6(0.3)$ & $4.7(0.3)^{b}$ & 0.149 \\
\hline Hemoglobin (g/dL) & $13.6(1.1)$ & $13.0(1.1)$ & $13.8(1.0)^{b}$ & $13.9(1.0)^{b}$ & $<0.0001$ \\
\hline Hematocrit $(\%)$ & $40.7(2.4)$ & $39.8(2.5)$ & $41.1(2.2)^{b}$ & $41.3(2.4)^{b}$ & 0.005 \\
\hline $\mathrm{MCV}(\mathrm{fL})$ & $88.4(3.9)$ & $87.6(4.1)$ & $88.8(4.1)$ & $88.8(3.5)$ & 0.231 \\
\hline $\mathrm{MCH}(\mathrm{pg})$ & $29.4(1.7)$ & $28.7(1.8)$ & $29.8(1.7)^{b}$ & $29.9(1.4)^{b}$ & 0.001 \\
\hline $\mathrm{MCHC}(\mathrm{g} / \mathrm{L})$ & $33.3(1.1)$ & $32.8(1.0)$ & $33.5(1.1)^{b}$ & $33.7(1.1)^{b}$ & $<0.0001$ \\
\hline RDW-CV (\%) & $12.9(0.9)$ & $13.1(0.9)$ & $12.8(0.8)$ & $12.7(0.9)$ & 0.341 \\
\hline $\mathrm{WBC}\left(\times 10^{9} / \mathrm{L}\right)$ & $6.4(1.3)$ & $6.5(1.3)$ & $6.6(1.3)$ & $6.3(1.6)$ & 0.390 \\
\hline Neutrophil $\left(\times 10^{9} / \mathrm{L}\right)$ & $3.4(1.4)$ & $3.5(1.4)$ & $3.4(1.5)$ & $3.2(1.4)$ & 0.493 \\
\hline Eosinophil $\left(\times 10^{9} / \mathrm{L}\right)$ & $0.13(0.5)$ & $0.12(0.5)$ & $0.15(0.5)$ & $0.12(0.5)$ & 0.377 \\
\hline Basophil $\left(\times 10^{9} / \mathrm{L}\right)$ & $0.03(0.06)$ & $0.02(0.06)$ & $0.03(0.05)$ & $0.02(0.06)$ & 0.075 \\
\hline Monocyte $\left(\times 10^{9} / \mathrm{L}\right)$ & $0.4(0.2)$ & $0.4(0.1)$ & $0.5(0.3)$ & $0.4(0.1)$ & 0.085 \\
\hline Lymphocyte $\left(\times 10^{9} / \mathrm{L}\right)$ & $2.4(0.7)$ & $2.3(0.6)$ & $2.5(0.7)$ & $2.3(0.8)$ & 0.606 \\
\hline Platelets $\left(\times 10^{9} / \mathrm{L}\right)$ & $248(56.9)$ & $267.9(62.7)$ & $242.9(54.4)^{b}$ & $234.4(48.4)^{b}$ & 0.019 \\
\hline \multicolumn{6}{|l|}{ TA duplication in $U G T 1 A 1$ gene } \\
\hline$[=]+[=][\%,(\mathrm{n})]$ & $48.8(71)$ & $67.3(33)$ & $53.1(26)^{b}$ & $25.0(12)^{b, c}$ & \\
\hline$[=]+[$ c.-41_-40dupTA $][\%,(\mathrm{n})]$ & $40.8(59)$ & $33.3(16)$ & $43.1(21)^{b}$ & $45.8(22)^{b}$ & $<0.0001$ \\
\hline c.-41_-40dupTA $[\%,(\mathrm{n})]$ & $10.9(16)$ & $0.0(0)$ & $3.9(2)^{b}$ & $29.2(14)^{b, c}$ & \\
\hline \multicolumn{6}{|c|}{$\begin{array}{l}\text { Data are presented as mean (standard deviation) for continuous variables and as percentage (number) for categorical variables. } \\
a P \text { value for } 1 \text {-way analysis of variance or } \chi^{2} \text { tests. }\end{array}$} \\
\hline
\end{tabular}

homozygosity; $\beta=0.381, P<0.0001$ when in heterozygosity), followed by $\mathrm{Hb}(\beta=0.297, P<0.0001)$, fasting hours $(\beta=$ $0.292, P<0.0001)$ and BMI $(\beta=-0.235, P=0.004)$.

\section{DISCUSSION}

Several studies aimed to clarify the influence of genetic and nongenetic factors in bilirubin levels..$^{2,3,21-27}$ Most of them

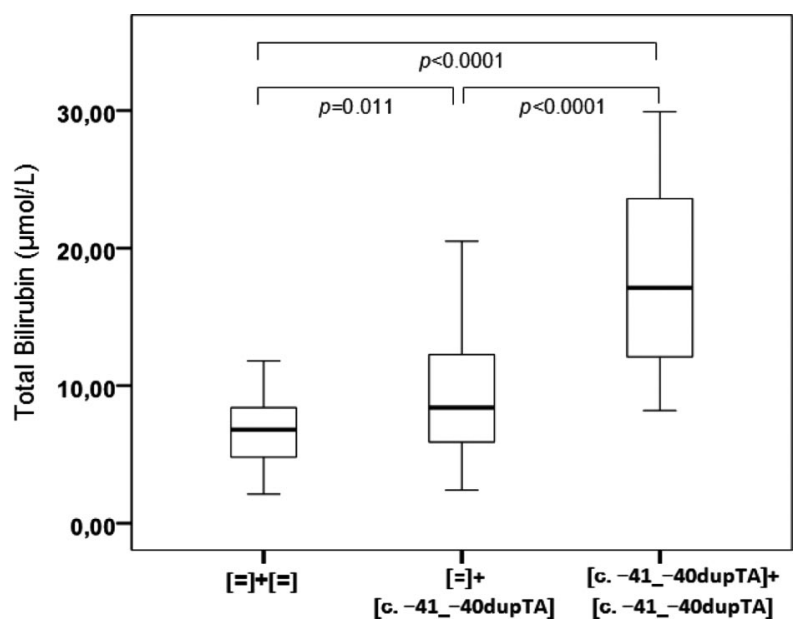

FIGURE 1. Total bilirubin plasma levels according to UGT1A1 genotype. focused only on genetic or nongenetic factors and included individuals of both sex and with a wide range of ages. In this work, we analyzed a cohort of young women with narrow age range to eliminate the potential interference of age and sex.

Our results showed that TA duplication in the promoter region of UGT1A1 gene, $\mathrm{Hb}$ concentration, fasting time and $\mathrm{BMI}$ are the main determinants of bilirubin levels.

According to bilirubin tertiles, our population showed an increase in bilirubin concentration that follows the genetic polymorphism present at the promoter region of the UGT1A1 gene. In fact, we found a higher prevalence of c.-41_-40dupTA allele in subjects with the highest bilirubin concentrations. Moreover, when the population was stratified by UGT1A1 genotype, we found significant differences for bilirubin levels between the 3 different genotypes (Figure 1), showing a strong association between higher bilirubin levels and the presence of TA duplication in the promoter region of UGT1A1 gene. This finding is in accordance with published data that defined the c.-41_-40dupTA allele as the most important cause of hyperbilirubinemia in white population. ${ }^{6,27}$

Because most of plasma bilirubin results from the catabolism of $\mathrm{Hb}$ in the macrophages from the reticuloendothelial system, it is expected that bilirubin concentration will increase as $\mathrm{Hb}$ concentration increases. Our results showed that $\mathrm{Hb}$, $\mathrm{MCH}$ and $\mathrm{MCHC}$ were increased in women with bilirubin levels $>6 \mu \mathrm{mol} / \mathrm{L}$ (second and third tertile). Moreover, we found positive and significant correlations between bilirubin levels and $\mathrm{Hb}, \mathrm{Ht}, \mathrm{MCH}$ and $\mathrm{MCHC}$. These results strongly suggest that red cell mass and $\mathrm{Hb}$ concentration are associated 
TABLE 2. Data from physical activity, smoking habits, oral contraceptive therapy, body fat, alcohol ingestion, fasting time, caloric intake distribution and body mass index by tertiles of total bilirubin in all population

\begin{tabular}{|c|c|c|c|c|c|}
\hline & \multirow{2}{*}{$\begin{array}{l}\text { Participants } \\
(\mathrm{n}=135)^{a}\end{array}$} & \multicolumn{4}{|c|}{ Tertiles of total bilirubin levels $(\mu \mathrm{mol} / \mathrm{L})$} \\
\hline & & $\mathrm{T} 1(\leq 6.0, \mathrm{n}=45)$ & $\mathrm{T} 2(6.0-9.2, \mathrm{n}=44)$ & $\mathrm{T} 3(\geq 9.2, \mathrm{n}=46)$ & $P^{a}$ \\
\hline \multicolumn{6}{|l|}{ Physical activity } \\
\hline Low $[\%,(n)]$ & $14.8(20)$ & $20.0(9)$ & $10.9(4)$ & $14.3(7)$ & \\
\hline Moderate $[\%,(n)]$ & $55.6(75)$ & $48.9(21)$ & $56.5(26)$ & $61.9(28)$ & 0.470 \\
\hline $\operatorname{High}[\%,(\mathrm{n})]$ & $29.6(40)$ & $33.3(15)$ & $32.6(14)$ & $23.8(11)$ & \\
\hline Smokers $[\%,(n)]$ & $11.9(16)$ & $14.9(7)$ & $13.0(6)$ & $7.1(3)$ & 0.504 \\
\hline With OCT $[\%,(n)]$ & $42.2(56)$ & $48.9(23)$ & $35.6(16)$ & $35.4(17)$ & 0.420 \\
\hline BMI $\left(\mathrm{kg} / \mathrm{m}^{2}\right)$ & $22.0(4.3)$ & $22.6(4.4)$ & $22.8(5.4)$ & $20.8(2.2)^{b}$ & 0.099 \\
\hline Body fat $(\%)$ & $25.7(6.9)$ & $27.4(7.7)$ & $26.1(7.2)$ & $24.3(5.7)$ & 0.211 \\
\hline Alcohol intake (mg/d) & $4.0(6.6)$ & $5.3(9.2)$ & $3.7(5.0)$ & $2.9(4.2)$ & 0.248 \\
\hline Fasting time $(\mathrm{hr})$ & $11.1(2.0)$ & $11.0(2.1)$ & $11.0(2.0)$ & $11.4(1.6)$ & 0.492 \\
\hline
\end{tabular}

with interindividual variations in bilirubin levels. This finding was previously reported in a study with patients with GS ${ }^{18}$; our study reports for the first time, in a young female population, an increase in $\mathrm{Hb}$ concentration that follows bilirubin levels (bilirubin $\geq 6 \mu \mathrm{mol} / \mathrm{L}$ ). We also found lower platelet counts in subjects with bilirubin levels higher than $6 \mu \mathrm{mol} / \mathrm{L}$. Low $\mathrm{Hb}$ concentration has been associated with an increased risk for cardiovascular disease events and mortality in patients with end-stage renal disease, ${ }^{28}$ and to heart failure ${ }^{29}$ in the general population. ${ }^{31}$ We doubt whether lower levels of bilirubin that probably occur in these cases represent an additional risk, because bilirubin may have an important role in the prevention of cardiovascular diseases, ${ }^{31,32}$ as a result of its antioxidant effect. ${ }^{31}$

We also found that fasting time is an independent variable associated with total bilirubin concentration. This association has been previously reported in subjects who were not stratified according to UGT1A1 genotype. ${ }^{24,25}$ The increase of bilirubin levels with fasting time seems to result from a decrease of the intestinal motility and elimination of bile pigments, causing bilirubin accumulation in the intestine-leading to an increased reflux of bilirubin into the plasma. ${ }^{33}$ In fasting subjects with a normal capacity of bilirubin conjugation, the accumulation of bilirubin in the intestine can be higher compared with those with a low capacity of bilirubin conjugation because of UGT1A1 polymorphisms, explaining the higher bilirubin levels found in women with normal number of TA repeats and with UGT1A1 polymorphisms, with the same fasting time.

Multivariate regression analysis also showed BMI as an independent variable associated with total bilirubin concentration. No significant differences were established for body fat between the 3 tertiles of total bilirubin levels; however, a trend for lower values was observed from the lowest to the highest bilirubin tertile. An inverse correlation between body fat and BMI with bilirubin concentration has been reported, ${ }^{34,35}$ but this association is still poorly clarified.

Regarding the influence of lifestyle variables, data from literature are still controversial. ${ }^{21,22,26} \mathrm{We}$ also analyzed the effect of other nongenetic factors in bilirubin levels, such as physical activity, tobacco smoking, alcohol intake and oral contraceptive therapy. We did not find any association between these factors and the changes in bilirubin concentration. This can be due to the characteristics of our sample, which, for instance, included only young women who did not have strong habits of smoking or did exceed the recommended limit of alcohol ingestion (data not shown).

In conclusion, we studied the effect of several factors that could contribute for total bilirubin concentration. We found that $\mathrm{Hb}$ concentration, fasting time and BMI are independent variables significantly associated with total bilirubin concentration in a female population. In the same way, total bilirubin concentration was strongly affected by the polymorphism presented in the promoter region of the UGTIAl gene. Further studies must be performed to determine the effect of these factors in different ages, in both men and women, and determine whether UGT1A1 variations, other than the TA duplication in the promoter region, could affect the total bilirubin levels.

\section{REFERENCES}

1. Bosma PJ, Seppen J, Goldhoor B, et al. Bilirubin UDP-glucuronosyltransferase 1 is the only relevant bilirubin glucuronidating isoform in man. J Biol Chem 1994;269:17960-4.

2. Strassburg CP, Kalthoff S, Ehmer U. Variability and function of family 1 uridine-5'-diphosphate glucuronosyltransferases (UGT1A). Crit Rev Clin Lab Sci 2008;45:485-530.

3. Hong AL, Huo D, Kim HJ, et al. UDP-glucuronosyltransferase 1A1 gene polymorphisms and total bilirubin levels in an ethnically diverse cohort of women. Drug Metab Dispos 2007;35:1254-61.

4. Costa E. Hematologically important mutations: bilirubin UDP-glucuronosyltransferase gene mutations in Gilbert and Crigler-Najjar syndromes. Blood Cell Mol Dis 2006;36:77-80.

5. Bosma PJ, Chowdhury JR, Bakker C, et al. The genetic basis of the reduced expression of bilirubin UDP-glucuronosyltransferase 1 in Gilbert's syndrome. N Engl J Med 1995;333:1171-5.

6. Clementi M, Di Gianantonio E, Fabris $\mathbf{L}$, et al. Inheritance of hyperbilirubinemia: evidence for a major autosomal recessive gene. Dig Liver Dis 2007;39:351-5.

7. Teich N, Lehmann I, Rosendahl J, et al. The inverse starving test is 
not a suitable provocation test for Gilbert's syndrome. BMC Res Notes 2008; $1: 35$.

8. Ideo G, De Franchis R, Del Ninno E, et al. Ethanol increases liver uridine-diphosphate-glucuronyltransferase. Experientia 1971;27:24-5.

9. Ritter JK, Kessler FK, Thompson ET, et al. Expression and inducibility of the human bilirubin UDP-glucuronosyltransferase UGT1A1 in liver and cultured primary hepatocytes: evidence for both genetic and environmental influences. Hepatology 1999;30:476-84.

10. Kanou M, Usui T, Ueyama H, et al. Stimulation of transcriptional expression of human UDP-glucuronosyltransferase 1A1 by dexamethasone. Mol Biol Rep 2004;31:151-8.

11. Ramirez J, Komoroski BJ, Mirkov S, et al. Study of the genetic determinants of UGT1A1 inducibility by phenobarbital in cultured human hepatocytes. Pharmacogenet Genomics 2006;16:79-86.

12. Hsieh TY, Shiu TY, Huang SM, et al. Molecular pathogenesis of Gilbert's syndrome: decreased TATA-binding protein binding affinity of UGT1A1 gene promoter. Pharmacogenet Genomics 2007;17:229-36.

13. Assenat $\mathbf{E}$, Gerbal-Chaloin $\mathbf{S}$, Larrey $\mathbf{D}$, et al. Interleukin $1 \beta$ inhibits CAR-induced expression of hepatic genes involved in drug and bilirubin clearance. Hepatology 2004;40:951-60.

14. Richardson T, Sherman M, Kalman D, et al. Expression of UDPglucuronosyltransferase isoform mRNAs during inflammation and infection in mouse liver and kidney. Drug Metab Dispos 2006;34:351-3.

15. Shapiro SM. Definition of the clinical spectrum of kernicterus and bilirubin-induced neurologic dysfunction (BIND). J Perinatol 2005;25: $54-9$.

16. Gullu H, Erdogan D, Tok D, et al. High serum bilirubin concentrations preserve coronary flow reserve and coronary microvascular functions. Arterioscler Thromb Vasc Biol 2005;25:2289-94.

17. Yesilova Z, Serdar M, Ercin CN, et al. Decreased oxidation susceptibility of plasma low density lipoproteins in patients with Gilbert's syndrome. J Gastroenterol Hepatol 2008;23:1556-60.

18. Buyukasik Y, Akman U, Buyukasik NS, et al. Evidence for higher red blood cell mass in persons with unconjugated hyperbilirubinemia and Gilbert's syndrome. Am J Med Sci 2008;335:115-9.

19. Guidelines for data processing and analysis of the International Physical Activity Questionnaire (IPAQ). Available at: http://www.ipaq.ki.se/ scoring.pdf. Accessed October 8, 2010.

20. Bancroft D, Kreamer B, Gourley R. Gilbert syndrome accelerates development of neonatal jaundice. J Pediatr 1998;132:656-60.

21. Van Hoydonck PG, Temme EH, Schouten EG. Serum bilirubin concentration in a Belgian population: the association with smoking status and type of cigarettes. Int J Epidemiol 2001;30:1465-72.

22. Chan-Yeung M, Ferreira P, Frohlich J, et al. The effects of age, smoking, and alcohol in routine laboratory tests. Am J Clin Pathol 1981;75:320-6.

23. Rosenthal P, Pincus M, Fink D. Sex- and Age-Related Differences in Bilirubin Concentrations in Serum. Clin Chem 1984;30:1382-6.

24. White GL, Nelson JA, Pedersen DM, et al. Fasting and gender (and altitude?) influence reference intervals for serum bilirubin in healthy adults. Clin Chem 1981;27:1140-2.

25. Winkel P, Statland BE, Bokelund H. Factors contributing to intraindividual variation of serum constituents: 5 . Short-term day-to-day and within-hour variation of serum constituents in healthy subjects. Clin Chem 1974;20:1520-7.

26. Ebele J, Emeka N, Ignatius M, et al. Effects of duration of use of hormonal contraceptives on liver function. Res J Med Sci 2009;3:52-5.

27. Beutler ET, Gelbart T, Demina A. Racial variability in the UDPglucuronosyltransferase 1 (UGT1A1) promoter: a balanced polymorphism for regulation of bilirubin metabolism? Proc Natl Acad Sci USA 1998;95:8170-4.

28. Foley RN, Parfrey PS, Harnett JD, et al. The impact of anemia on cardiomyopathy, morbidity and mortality in end-stage renal disease. Am J Kidney Dis 1996;28:53-61.

29. Horwich TB, Fonarow GC, Hamilton MA, et al. Anemia is associated with worse symptoms, greater impairment in functional capacity and a significant increase in mortality in patients with advanced heart failure. J Am Coll Cardiol 2002;39:1780-6.

30. Culleton BF, Manns BJ, Zhang J, et al. Impact of anemia on hospitalization and mortality in older adults. Blood 2006;107:38:41-6.

31. Schwertner HA, Libor V. Gilbert syndrome, UGT1A1*28 allele, and cardiovascular disease risk: possible protective effects and therapeutic applications of bilirubin. Atherosclerosis 2008;198:1-11.

32. Troughton J, Woodside J, Young IS, et al; PRIME Study Group. Bilirubin and coronary heart disease risk in the Prospective Epidemiological Study of Myocardial Infarction (PRIME). Eur J Cardiovasc Prev Rehabil 2007;142:79-84.

33. Goresky CA, Gordon ER, Shaffer EA, et al. Definition of a conjugation of dysfunction in Gilbert's syndrome: studies of the handling of bilirubin loads and of the pattern of bilirubin conjugates secreted in bile. Clin Sci Mol Med 1978;55:63-71.

34. Hopkins PN, Wu LL, Hunt SC. Higher serum bilirubin is associated with decreased risk for early familial coronary artery disease. Arterioscler Thromb Vasc Biol 1996;16:250-5.

35. Ohnakaa K, Konob $\mathbf{S}$, Inoguchic $\mathbf{T}$, et al. Inverse associations of serum bilirubin with high sensitivity C-reactive protein, glycated hemoglobin, and prevalence of type 2 diabetes in middle-aged and elderly Japanese men and women. Diab Res Clin Pract 2010;88:103-10. 


\section{AUTHOR QUERIES}

\section{AUTHOR PLEASE ANSWER ALL QUERIES}

1-Kindly confirm whether the short title is OK as given.

2 -Per journal style, unstructured abstracts should be $\leq 150$ words. Kindly modify the abstract to match the word limit.

3-Please check whether the term "Plasmtic total bilirubin" is OK as given.

4 -Please confirm whether location for the manufacturer "SPSS" is OK as given.

5-Please spell out HSD.

6-Please provide the significance of the bold values in Table 1.

7-Please note that Ref. 30 is uncited. Kindly cite the same sequentially.

8-Please check whether affiliations are OK as typeset and provide department/division name (if any) for all affiliations. 